\title{
Epidural anaesthesia in a patient with advanced multiple sclerosis
}

Inês Ferraz, Francisca Tavares, Joana Afonso, Maria João Tarroso

\section{INTRODUCTION}

Multiple sclerosis is the most common demyelinating disease of the central nervous system. It's more frequent in women, but the prognosis is worst in men. Perioperative physiological stress and anaesthesia may exacerbate the disease. We present a case report of an epidural anaesthesia in a patient with advanced multiple sclerosis.

\section{Case Report:}

A 46 year-old male patient with advanced multiple sclerosis, was scheduled to elective sacral ulcer flap reconstruction. He had a history of restrictive pulmonary disease, severe dysphagia and tetraparesis.

The day before and in the morning of the surgery, he received $10 \mathrm{mg}$ of metoclopramide and $50 \mathrm{mg}$ of ranitidine. In the operating room, standard monitorization and supplementary oxygenation $\left(\mathrm{FiO}_{2}\right.$ of 0,4$)$ was started. Patient was preloaded with $500 \mathrm{ml}$ of crystalloid fluid. Epidural anaesthesia was performed with $37,5 \mathrm{mg}$ of ropivacaine $(0.75 \%)$ and $0,02 \mathrm{mg}$ of sufentanil. Prone position with protection of pressure areas and active warming were accomplished. The surgery lasted one hour and was uneventful. The patient was discharged home and neurologic exacerbations were not reported.

\section{Discussion}

Multiple sclerosis has many anaesthetic implications, so it is crucial to ponder the advantages and disadvantages of each option, taking into account the patient state and the type of surgery. In this case, we decide to perform neuroaxial anaesthesia in order to avoid pulmonary aspiration and respiratory complications. Epidural block is a safe option with less risk of neurotoxicity comparing with subarachnoid block. Hemodynamic stability, normothermia, absence of technical difficulties, low doses of local anaesthetics and suitable pain control, were important contributing factors for the good outcome.

\section{Learning points}

- Epidural block is a safe technique in patients with multiple sclerosis disease.

- Maintenance of hemodynamic stability, normothermia, respiratory function and suitable pain control are crucial in these patients 